\title{
Correction to: Transcranial Magneto-Acoustic Stimulation Improves Neuroplasticity in Hippocampus of Parkinson's Disease Model Mice
}

Yuexiang Wang ${ }^{1} \cdot$ Lina Feng $^{1} \cdot$ Shikun $\mathrm{Liu}^{2} \cdot$ Xiaoqing Zhou ${ }^{2} \cdot$ Tao Yin $^{2} \cdot$ Zhipeng Liu $^{2} \cdot$ Zhuo Yang $^{1}$

Published online: 9 July 2019

(C) The American Society for Experimental NeuroTherapeutics, Inc. 2019

\section{Correction to: Neurotherapeutics}

https://doi.org/10.1007/s13311-019-00732-5

The authors would like to correct X. Zhou's grant number from the National Natural Science Foundation of China to 81601633 .

Publisher's Note Springer Nature remains neutral with regard to jurisdictional claims in published maps and institutional affiliations.

The online version of the original article can be found at https://oi.org/ 10.1007/s13311-019-00732-5

$\triangle$ Zhipeng Liu

lzpeng67@163.com

$\triangle$ Zhuo Yang

zhuoyang@nankai.edu.cn

1 College of Medicine, State Key Laboratory of Medicinal Chemical Biology, Tianjin Key Laboratory of Tumor Microenvironment and Neurovascular Regulation, Nankai University, Tianjin 300071, China

2 Institute of Biomedical Engineering, Chinese Academy of Medical Sciences \& Peking Union Medical College, Tianjin 300192, China 